\title{
Higher Mahler measures and zeta functions
}

by

N. Kurokawa (Tokyo), M. Lalín (Edmonton) and H. Ochiai (Nagoya)

1. Introduction. The (logarithmic) Mahler measure of a non-zero Laurent polynomial $P \in \mathbb{C}\left[x_{1}^{ \pm 1}, \ldots, x_{n}^{ \pm 1}\right]$ is defined by

$$
m(P)=\int_{0}^{1} \cdots \int_{0}^{1} \log \left|P\left(e^{2 \pi i \theta_{1}}, \ldots, e^{2 \pi i \theta_{n}}\right)\right| d \theta_{1} \cdots d \theta_{n} .
$$

In this work, we consider the following generalization:

Definition 1. The $k$-higher Mahler measure of $P$ is defined by

$$
m_{k}(P):=\int_{0}^{1} \cdots \int_{0}^{1} \log ^{k}\left|P\left(e^{2 \pi i \theta_{1}}, \ldots, e^{2 \pi i \theta_{n}}\right)\right| d \theta_{1} \cdots d \theta_{n} .
$$

In particular,

$$
m_{1}(P)=m(P) \quad \text { and } \quad m_{0}(P)=1 .
$$

These terms are the coefficients in the Taylor expansion of Akatsuka's zeta Mahler measure

$$
Z(s, P)=\int_{0}^{1} \cdots \int_{0}^{1}\left|P\left(e^{2 \pi i \theta_{1}}, \ldots, e^{2 \pi i \theta_{n}}\right)\right|^{s} d \theta_{1} \cdots d \theta_{n},
$$

that is,

$$
Z(s, P)=\sum_{k=0}^{\infty} \frac{m_{k}(P) s^{k}}{k !} .
$$

Akatsuka [1] computed the zeta Mahler measure $Z(s, x-c)$ for a constant $c$.

A natural generalization for the $k$-higher Mahler measure is the multiple higher Mahler measure for more than one polynomial.

2000 Mathematics Subject Classification: 11M06, $11 \mathrm{R} 09$.

Key words and phrases: Mahler measure, zeta functions, Dirichlet $L$-functions, polylogarithms.

Research of M. Lalín supported by University of Alberta Fac. Sci. Startup Grant N031000610 and NSERC Discovery Grant 355412-2008. 
Definition 2. Let $P_{1}, \ldots, P_{l} \in \mathbb{C}\left[x_{1}^{ \pm 1}, \ldots, x_{r}^{ \pm 1}\right]$ be non-zero Laurent polynomials. Their multiple higher Mahler measure is defined by $m\left(P_{1}, \ldots, P_{l}\right)$ $:=\int_{0}^{1} \cdots \int_{0}^{1}\left(\log \left|P_{1}\left(e^{2 \pi i \theta_{1}}, \ldots, e^{2 \pi i \theta_{r}}\right)\right|\right) \cdots\left(\log \left|P_{l}\left(e^{2 \pi i \theta_{1}}, \ldots, e^{2 \pi i \theta_{r}}\right)\right|\right) d \theta_{1} \cdots d \theta_{r}$.

This construction yields the higher Mahler measures of one polynomial as a special case:

$$
m_{k}(P)=m(\underbrace{P, \ldots, P}_{k}) .
$$

Moreover, the above definition implies that

$$
m\left(P_{1}\right) \cdots m\left(P_{l}\right)=m\left(P_{1}, \ldots, P_{l}\right)
$$

when the variables of $P_{j}$ 's on the right-hand side are algebraically independent. This identity leads us to speculate about a product structure for the logarithmic Mahler measure. This would be a novel property, since the logarithmic Mahler measure is known to be additive, but no multiplicative structure is known.

This definition has a natural counterpart in the world of zeta Mahler measures, namely, the higher zeta Mahler measure defined by

$$
\begin{aligned}
& Z\left(s_{1}, \ldots, s_{l} ; P_{1}, \ldots, P_{l}\right) \\
& \quad=\int_{0}^{1} \cdots \int_{0}^{1}\left|P_{1}\left(e^{2 \pi i \theta_{1}}, \ldots, e^{2 \pi i \theta_{r}}\right)\right|^{s_{1}} \cdots\left|P_{l}\left(e^{2 \pi i \theta_{1}}, \ldots, e^{2 \pi i \theta_{r}}\right)\right|^{s_{l}} d \theta_{1} \cdots d \theta_{r} .
\end{aligned}
$$

Its Taylor coefficients are related to the multiple higher Mahler measure:

$$
\frac{\partial^{l}}{\partial s_{1} \cdots \partial s_{l}} Z\left(0, \ldots, 0 ; P_{1}, \ldots, P_{l}\right)=m\left(P_{1}, \ldots, P_{l}\right) .
$$

In this work, we compute the simplest examples of these measures and explore their basic properties. In Section 2 we consider the higher Mahler measure for one-variable polynomials. More precisely, we consider linear polynomials in one variable. In particular, we obtain

$$
\begin{aligned}
m_{2}(x-1) & =\frac{\pi^{2}}{12}, \\
m_{3}(x-1) & =-\frac{3 \zeta(3)}{2}, \\
m_{4}(x-1) & =\frac{19 \pi^{4}}{240}, \\
m\left(1-x, 1-e^{2 \pi i \alpha} x\right) & =\frac{\pi^{2}}{2}\left(\alpha^{2}-\alpha+\frac{1}{6}\right), \quad 0 \leq \alpha \leq 1 .
\end{aligned}
$$


In Section 3, we consider two examples of two-variable Mahler measure and we compute $m_{2}$. Sections 4 and 5 deal with examples of zeta Mahler measures of linear polynomials and their applications to the computation of higher Mahler measure, recovering the results from Section 2 and giving an insight into them. Finally, we explore harder examples of zeta and higher Mahler measures in Section 6. For example,

$$
\begin{aligned}
m_{2}(x+y+2) & =\frac{\zeta(2)}{2} \\
m_{3}(x+y+2) & =\frac{9}{2} \log 2 \zeta(2)-\frac{15}{4} \zeta(3), \\
Z\left(s, x+x^{-1}+y+y^{-1}+c\right) & =c^{s}{ }_{3} F_{2}\left(\begin{array}{c}
-\frac{s}{2}, \frac{1-s}{2}, \frac{1}{2} \\
1,1
\end{array} \mid \frac{16}{c^{2}}\right), \quad c>4 .
\end{aligned}
$$

\section{Higher Mahler measure of one-variable polynomials}

2.1. The case of $1-x$. Our first example is given by the simplest possible polynomial, namely $P=1-x$.

THEOREM 3.

$$
m_{k}(1-x)=\sum_{b_{1}+\cdots+b_{h}=k, b_{i} \geq 2} \frac{(-1)^{k} k !}{2^{2 h}} \zeta\left(b_{1}, \ldots, b_{h}\right),
$$

where $\zeta\left(b_{1}, \ldots, b_{h}\right)$ denotes a multizeta value, i.e.,

$$
\zeta\left(b_{1}, \ldots, b_{h}\right)=\sum_{l_{1}<\cdots<l_{h}} \frac{1}{l_{1}^{b_{1}} \cdots l_{h}^{b_{h}}} .
$$

The right-hand side of the formula in Theorem 3 can be rewritten in terms of classical zeta values by using the following result.

Proposition 4.

$$
\begin{aligned}
\sum_{\sigma \in S_{h}} \zeta\left(b_{\sigma(1)}, \ldots, b_{\sigma(h)}\right) & \\
& =\sum_{e_{1}+\cdots+e_{l}=h}(-1)^{h-l} \prod_{s=1}^{l}\left(e_{s}-1\right) ! \sum \zeta\left(\sum_{k \in \pi_{1}} b_{k}\right) \cdots \zeta\left(\sum_{k \in \pi_{l}} b_{k}\right),
\end{aligned}
$$

where the sum on the right is taken over all the possible unordered partitions of the set $\{1, \ldots, h\}$ into $l$ subsets $\pi_{1}, \ldots, \pi_{l}$ with $e_{1}, \ldots, e_{l}$ elements respectively.

Proof of Theorem 3. First observe that $x$ varies in the unit circle. Therefore, we can choose the principal branch for the logarithm. We proceed to 
write the function in terms of integrals of rational functions. We have

$$
\begin{aligned}
\log ^{k}|1-x| & =(\Re \log (1-x))^{k}=\left(\frac{1}{2}\left(\log (1-x)+\log \left(1-x^{-1}\right)\right)\right)^{k} \\
& =\frac{1}{2^{k}}\left(\int_{0}^{1} \frac{d t}{t-x^{-1}}+\int_{0}^{1} \frac{d t}{t-x}\right)^{k}=\frac{1}{2^{k}} \sum_{j=0}^{k}\left(\begin{array}{c}
k \\
j
\end{array}\right)\left(\int_{0}^{1} \frac{d t}{t-x^{-1}}\right)^{j}\left(\int_{0}^{1} \frac{d t}{t-x}\right)^{k-j} .
\end{aligned}
$$

Now observe that

$$
\begin{aligned}
& \left(\int_{0}^{1} \frac{d t}{t-x^{-1}}\right)^{j}\left(\int_{0}^{1} \frac{d t}{t-x}\right)^{k-j} \\
& \quad=j !(k-j) ! \int_{0}^{1} \underbrace{\frac{d t}{t-x^{-1}} \circ \cdots \circ \frac{d t}{t-x^{-1}}}_{j} \int_{0}^{1} \underbrace{\frac{d t}{t-x} \circ \cdots \circ \frac{d t}{t-x}}_{k-j} .
\end{aligned}
$$

We have just used the iterated integral notation for hyperlogarithms.

Combining the previous equalities gives

$$
\begin{aligned}
m_{k}(1-x) & =\frac{1}{2 \pi i} \int_{|x|=1} \log ^{k}|1-x| \frac{d x}{x} \\
& =\frac{k !}{2^{k}} \sum_{j=0}^{k} \frac{1}{2 \pi i} \int_{|x|=1} \int_{0}^{1} \underbrace{\frac{d t}{t-x^{-1}} \circ \cdots \circ \frac{d t}{t-x^{-1}}}_{j} \int_{0}^{1} \underbrace{\frac{d t}{t-x} \circ \cdots \circ \frac{d t}{t-x}}_{k-j} \frac{d x}{x} .
\end{aligned}
$$

If we now set $s=x t$ in the first $j$-fold integral and $s=t / x$ in the second $(k-j)$-fold integral, the above becomes

$$
\frac{k !}{2^{k}} \sum_{j=0}^{k} \frac{1}{2 \pi i} \int_{|x|=1} \int_{0}^{x} \frac{d s}{s-1} \circ \cdots \circ \frac{d s}{s-1} \int_{0}^{x^{-1}} \frac{d s}{s-1} \circ \cdots \circ \frac{d s}{s-1} \frac{d x}{x} .
$$

We proceed to compute the integrals in terms of multiple polylogarithms:

$$
\begin{aligned}
m_{k}(1-x) & =\frac{(-1)^{k} k !}{2^{k}} \sum_{j=0}^{k} \frac{1}{2 \pi i} \int_{|x|=1}\left(\sum_{\substack{0<l_{1}<\cdots<l_{j}<\infty \\
0<m_{1}<\cdots<m_{k-j}<\infty}} \frac{x^{l_{j}-m_{k-j}}}{l_{1} \cdots l_{j} m_{1} \cdots m_{k-j}}\right) \frac{d x}{x} \\
= & \frac{(-1)^{k} k !}{2^{k}} \sum_{j=1}^{k-1} \sum_{\substack{0<l_{1}<\cdots<l_{j-1}<u<\infty \\
0<m_{1}<\cdots<m_{k-j-1}<u<\infty}} \frac{1}{l_{1} \cdots l_{j-1} m_{1} \cdots m_{k-j-1} u^{2}} .
\end{aligned}
$$

Now we need to analyze each term of the form

$$
\sum_{\substack{0<l_{1}<\cdots<l_{j-1}<u<\infty \\ 0<m_{1}<\cdots<m_{k-j-1}<u<\infty}} \frac{1}{l_{1} \cdots l_{j-1} m_{1} \cdots m_{k-j-1} u^{2}} .
$$


For an $h$-tuple $a_{1}, \ldots, a_{h}$ such that $a_{1}+\cdots+a_{h}=k-2 h$, we set

$$
d_{a_{1}, \ldots, a_{h}}=\sum_{e_{1}+\cdots+e_{h}=j-h}\left(\begin{array}{l}
a_{1} \\
e_{1}
\end{array}\right) \cdots\left(\begin{array}{c}
a_{h} \\
e_{h}
\end{array}\right)=\left(\begin{array}{c}
a_{1}+\cdots+a_{h} \\
e_{1}+\cdots+e_{h}
\end{array}\right)=\left(\begin{array}{c}
k-2 h \\
j-h
\end{array}\right) .
$$

Then the term (1) is equal to

$$
\sum_{h=1}^{\min \{j-1, k-j-1\}} d_{a_{1}, \ldots, a_{h}} \zeta\left(\{1\}_{a_{1}}, 2, \ldots,\{1\}_{a_{h}}, 2\right) .
$$

Note that each term $\zeta\left(\{1\}_{a_{1}}, 2, \ldots,\{1\}_{a_{h}}, 2\right)$ comes from choosing $h-1$ of the l's and $h-1$ of the $m$ 's and making them equal in pairs. Once this has been done, one can choose the way the other l's and $m$ 's are ordered. All these choices give rise to the coefficients $d_{a_{1}, \ldots, a_{h}}$.

The total sum is given by

$$
m_{k}(1-x)=\sum_{h=1}^{k-1} c_{a_{1}, \ldots, a_{h}} \zeta\left(\{1\}_{a_{1}}, 2, \ldots,\{1\}_{a_{h}}, 2\right),
$$

where

$$
c_{a_{1}, \ldots, a_{h}}=\frac{(-1)^{k} k !}{2^{k}} \sum_{j=1}^{k-1}\left(\begin{array}{c}
k-2 h \\
j-h
\end{array}\right)=\frac{(-1)^{k} k !}{2^{k}} 2^{k-2 h}=\frac{(-1)^{k} k !}{2^{2 h}} .
$$

On the other hand,

$$
\zeta\left(\{1\}_{a_{1}}, 2, \ldots,\{1\}_{a_{h}}, 2\right)=\zeta\left(a_{h}+2, \ldots, a_{1}+2\right) .
$$

To see this well-known fact, observe that the term on the left is

$$
(-1)^{k-h} \int_{0}^{1} \underbrace{\frac{d t}{t-1} \circ \cdots \circ \frac{d t}{t-1}}_{a_{1}+1} \circ \frac{d t}{t} \circ \cdots \circ \underbrace{\frac{d t}{t-1} \circ \cdots \circ \frac{d t}{t-1}}_{a_{h}+1} \circ \frac{d t}{t} .
$$

Making the change $t \mapsto 1-t$ gives

$$
(-1)^{k-h}(-1)^{k} \int_{0}^{1} \frac{d t}{t-1} \circ \underbrace{\frac{d t}{t} \circ \cdots \circ \frac{d t}{t}}_{a_{h}+1} \circ \cdots \circ \frac{d t}{t-1} \circ \underbrace{\frac{d t}{t} \circ \cdots \circ \frac{d t}{t}}_{a_{1}+1},
$$

which corresponds to the term on the right. Thus, the total sum is

$$
m_{k}(1-x)=\sum_{b_{1}+\cdots+b_{h}=k, b_{i} \geq 2} \frac{(-1)^{k} k !}{2^{2 h}} \zeta\left(b_{1}, \ldots, b_{h}\right) .
$$

We give a proof of Proposition 4 for completeness.

Proof of Proposition 4. We first show that we can write

$$
\sum_{\sigma \in S_{h}} \zeta\left(b_{\sigma(1)}, \ldots, b_{\sigma(h)}\right)=\sum_{e_{1}+\cdots+e_{l}=h} r\left(e_{1}, \ldots, e_{l}\right) \sum \zeta\left(\sum_{k \in \pi_{1}} b_{k}\right) \cdots \zeta\left(\sum_{k \in \pi_{l}} b_{k}\right)
$$


where the function $r\left(e_{1}, \ldots, e_{l}\right)$ satisfies some recurrence relationships. Here, as in the statement, the sum on the right is taken over all possible unordered partitions of $\{1, \ldots, h\}$ into $l$ subsets $\pi_{1}, \ldots, \pi_{l}$ with $e_{1}, \ldots, e_{l}$ elements respectively.

Notice that $r$ is a function that is invariant under any permutation of its arguments. We proceed by induction on $h$. It is clear that $r(1)=1$. Also

$$
\zeta(a, b)+\zeta(b, a)=\zeta(a) \zeta(b)-\zeta(a+b),
$$

which yields $r(1,1)=1, r(2)=-1$.

Assume that the case of $h$ is settled. Now, we multiply everything by $\zeta\left(b_{h+1}\right)$,

$$
\begin{aligned}
\sum_{\sigma \in S_{h}} \zeta\left(b_{\sigma(1)}, \ldots, b_{\sigma(h)}\right) \zeta\left(b_{h+1}\right) & \\
= & \sum_{e_{1}+\cdots+e_{l}=h} r\left(e_{1}, \ldots, e_{l}\right) \sum \zeta\left(\sum_{k \in \pi_{1}} b_{k}\right) \cdots \zeta\left(\sum_{k \in \pi_{l}} b_{k}\right) \zeta\left(b_{h+1}\right) .
\end{aligned}
$$

Observe that

$$
\begin{aligned}
\sum_{\sigma \in S_{h}} \zeta\left(b_{\sigma(1)}, \ldots, b_{\sigma(h)}\right) \zeta\left(b_{h+1}\right)= & \sum_{\sigma \in S_{h+1}} \zeta\left(b_{\sigma(1)}, \ldots, b_{\sigma(h+1)}\right) \\
& +\sum_{j=1}^{h} \sum_{\sigma \in S_{h}} \zeta\left(b_{\sigma(1)}, \ldots, b_{\sigma(\breve{j})}, \ldots, b_{\sigma(h)}\right),
\end{aligned}
$$

where $b_{\breve{j}}=b_{j}+b_{h+1}$. Hence,

$$
\begin{aligned}
\sum_{\sigma \in S_{h+1}} \zeta\left(b_{\sigma(1)}, \ldots,\right. & \left.b_{\sigma(h+1)}\right) \\
= & \sum_{e_{1}+\cdots+e_{l}=h} r\left(e_{1}, \ldots, e_{l}\right) \sum \zeta\left(\sum_{k \in \pi_{1}} b_{k}\right) \cdots \zeta\left(\sum_{k \in \pi_{l}} b_{k}\right) \zeta\left(b_{h+1}\right) \\
& -\sum_{j=1}^{h} \sum_{e_{1}+\cdots+e_{l}=h} r\left(e_{1}, \ldots, e_{l}\right) \\
& \times \zeta\left(\sum_{k \in \pi_{1}} b_{k}\right) \cdots \zeta\left(b_{h+1}+\sum_{k \in \pi_{f}} b_{k}\right) \cdots \zeta\left(\sum_{k \in \pi_{l}} b_{k}\right) \zeta\left(b_{h+1}\right) .
\end{aligned}
$$

From the above equation, we deduce the following identities:

$$
\begin{gathered}
r\left(e_{1}, \ldots, e_{f}, 1, e_{f+1}, \ldots, e_{l}\right)=r\left(e_{1}, \ldots, e_{f}, e_{f+1}, \ldots, e_{l}\right), \\
r\left(e_{1}, \ldots, e_{f}+1, \ldots, e_{l}\right)=-e_{f} r\left(e_{1}, \ldots, e_{f}, \ldots, e_{l}\right) .
\end{gathered}
$$


Now it is very easy to conclude that

$$
r\left(e_{1}, \ldots, e_{l}\right)=(-1)^{h-l} \prod_{s=1}^{l}\left(e_{s}-1\right) !
$$

EXAMPLES 5 . Theorem 3 enables us to compute $m_{k}(1-x)$. Here are the first few examples for $k=2,3, \ldots, 6$ :

$$
\begin{aligned}
m_{2}(1-x)= & \zeta(2) \\
m_{3}(1-x)= & -6\left(\frac{\zeta(3)}{4}\right)=-\frac{3 \zeta(3)}{2} \\
m_{4}(1-x)= & 24\left(\frac{\zeta(4)}{4}+\frac{\zeta(2,2)}{16}\right) \\
= & 6 \zeta(4)+\frac{3\left(\zeta(2)^{2}-\zeta(4)\right)}{4}=\frac{3 \zeta(2)^{2}+21 \zeta(4)}{4} \\
m_{5}(1-x)= & -120\left(\frac{\zeta(5)}{4}+\frac{\zeta(2,3)+\zeta(3,2)}{16}\right) \\
= & -30 \zeta(5)-\frac{15(\zeta(2) \zeta(3)-\zeta(5))}{2}=-\frac{15 \zeta(2) \zeta(3)+45 \zeta(5)}{2} \\
m_{6}(1-x)= & 720\left(\frac{\zeta(6)}{4}+\frac{\zeta(3,3)}{16}+\frac{\zeta(2,4)+\zeta(4,2)}{16}+\frac{\zeta(2,2,2)}{64}\right) \\
= & 180 \zeta(6)+\frac{45\left(\zeta(3)^{2}-\zeta(6)\right)}{2}+45(\zeta(2) \zeta(4)-\zeta(6)) \\
& +\frac{45\left(2 \zeta(6)-3 \zeta(2) \zeta(4)+\zeta(2)^{3}\right)}{4 \cdot 6} \\
= & \frac{930 \zeta(6)+180 \zeta(3)^{2}+315 \zeta(2) \zeta(4)+15 \zeta(2)^{3}}{8} .
\end{aligned}
$$

Remark 6. Ohno and Zagier [3] prove a result that generalizes Proposition 4. Following their notation from [3, Theorem 1], and setting $y=0$, $z=x^{2} / 4$ (so that $s=n$ ), we have

$$
\sum_{k=2}^{\infty} \sum_{b_{1}+\cdots+b_{h}=k, b_{i} \geq 2} \frac{1}{2^{2 h}} \zeta\left(b_{1}, \ldots, b_{h}\right) x^{k}=\exp \left(\sum_{t=2}^{\infty} \frac{\zeta(t)}{t} x^{t}\left(1-\frac{1}{2^{t-1}}\right)\right) .
$$

This identity also explains the relationship between the result of Theorem 3 and the result that is recovered in Section 4.2.

2.2. Higher Mahler measure for several linear polynomials. As before, the simplest case to consider involves linear polynomials in one variable. 
TheOREM 7. For $0 \leq \alpha \leq 1$,

$$
m\left(1-x, 1-e^{2 \pi i \alpha} x\right)=\frac{\pi^{2}}{2}\left(\alpha^{2}-\alpha+\frac{1}{6}\right) .
$$

In particular, one obtains the following examples:

EXAMPLES 8.

$$
\begin{aligned}
m(1-x, 1-x) & =\frac{\pi^{2}}{12}, \\
m(1-x, 1+x) & =-\frac{\pi^{2}}{24}, \\
m(1-x, 1 \pm i x) & =-\frac{\pi^{2}}{96}, \\
m\left(1-x, 1-e^{2 \pi i \alpha} x\right) & =0 \Leftrightarrow \alpha=\frac{3 \pm \sqrt{3}}{6} .
\end{aligned}
$$

Proof of Theorem 7. By definition,

$$
\begin{aligned}
m\left(1-x, 1-e^{2 \pi i \alpha} x\right) & =\int_{0}^{1} \Re \log \left(1-e^{2 \pi i \theta}\right) \cdot \Re \log \left(1-e^{2 \pi i(\theta+\alpha)}\right) d \theta \\
& =\int_{0}^{1}\left(-\sum_{k=1}^{\infty} \frac{1}{k} \cos 2 \pi k \theta\right)\left(-\sum_{l=1}^{\infty} \frac{1}{l} \cos 2 \pi(\theta+\alpha)\right) d \theta \\
& =\sum_{k, l \geq 1} \frac{1}{k l} \int_{0}^{1} \cos (2 \pi k \theta) \cos (2 \pi l(\theta+\alpha)) d \theta .
\end{aligned}
$$

On the other hand,

$$
\int_{0}^{1} \cos (2 \pi k \theta) \cos (2 \pi l(\theta+\alpha)) d \theta= \begin{cases}\frac{1}{2} \cos 2 \pi k \alpha & \text { if } l=k \\ 0 & \text { otherwise. }\end{cases}
$$

By putting everything together we conclude that

$$
m\left(1-x, 1-e^{2 \pi i \alpha} x\right)=\frac{1}{2} \sum_{k=1}^{\infty} \frac{\cos 2 \pi k \alpha}{k^{2}}=\frac{\pi^{2}}{2}\left(\alpha^{2}-\alpha+\frac{1}{6}\right) .
$$

REMARK 9. The same calculation shows that

$$
m(1-\alpha x, 1-\beta x)= \begin{cases}\frac{1}{2} \Re \operatorname{Li}_{2}(\alpha \bar{\beta}) & \text { if }|\alpha|,|\beta| \leq 1, \\ \frac{1}{2} \Re \operatorname{Li}_{2}\left(\alpha \beta /|\alpha|^{2}\right) & \text { if }|\alpha| \geq 1,|\beta| \leq 1, \\ \frac{1}{2} \Re \operatorname{Li}_{2}\left(\alpha \bar{\beta} /|\alpha \beta|^{2}\right)+\log |\alpha| \log |\beta| & \text { if }|\alpha|,|\beta| \geq 1 .\end{cases}
$$


From this, one sees that for $P \in \mathbb{C}\left[x^{ \pm 1}\right], m_{2}(P)$ is a combination of dilogarithms and products of logarithms. In fact, for $P(x)=c x^{s} \prod_{j=1}^{r}\left(1-\alpha_{j} x\right)$, we have

$$
\begin{aligned}
m_{2}(P) & =m(P, P) \\
& =(\log |c|)^{2}+2(\log |c|) \sum_{j=1}^{r} \log ^{+}\left|\alpha_{j}\right|+\sum_{j, k=1}^{r} m\left(1-\alpha_{j} x, 1-\alpha_{k} x\right) .
\end{aligned}
$$

The formula above plays an analogous role to Jensen's formula.

REMARK 10. The previous computations may be extended to multiple higher Mahler measures involving more than two linear polynomials. For example,

$$
\begin{aligned}
m\left(1-x, 1-e^{2 \pi i \alpha} x, 1-e^{2 \pi i \beta} x\right)= & -\frac{1}{4} \sum_{k, l \geq 1} \frac{\cos 2 \pi((k+l) \beta-l \alpha)}{k l(k+l)} \\
& -\frac{1}{4} \sum_{k, m \geq 1} \frac{\cos 2 \pi((k+m) \alpha-m \beta)}{k m(k+m)} \\
& -\frac{1}{4} \sum_{l, m \geq 1} \frac{\cos 2 \pi(l \alpha+m \beta)}{l m(l+m)} .
\end{aligned}
$$

3. Higher Mahler measure of two-variable polynomials. In this section we are going to consider examples of higher Mahler measures of polynomials in two variables. In particular, we will focus on the computation of $m_{2}$ using the formula from Remark 9, analogously to the way Jensen's formula is applied for computing the classical Mahler measure of multivariable polynomials.

The two polynomials that we consider were among the first examples of multivariable polynomials to be computed in terms of Mahler measure (by Smyth [6]).

3.1. $m_{2}(x+y+1)$

THEOREM 11.

$$
m_{2}(x+y+1)=\frac{5 \pi^{2}}{54} .
$$

Proof. We have, by definition,

$$
m_{2}(x+y+1)=\frac{1}{(2 \pi i)^{2}} \int_{|y|=1} \int_{|x|=1} \log ^{2}|x+y+1| \frac{d x}{x} \frac{d y}{y} .
$$


By the result from Remark 9 with respect to the variable $y$,

$$
\begin{aligned}
m_{2}(x+y+1)= & \frac{1}{2 \pi i} \int_{|x|=1,|x+1| \leq 1} \frac{1}{2} \operatorname{Li}_{2}\left(|1+x|^{2}\right) \frac{d x}{x} \\
& +\frac{1}{2 \pi i} \int_{|x|=1,|x+1| \geq 1}\left(\frac{1}{2} \operatorname{Li}_{2}\left(\frac{1}{|1+x|^{2}}\right)+\log ^{2}|1+x|\right) \frac{d x}{x} .
\end{aligned}
$$

Recalling the functional identity for the dilogarithm,

$$
\operatorname{Li}_{2}(z)=-\mathrm{Li}_{2}\left(\frac{1}{z}\right)-\frac{1}{2} \log ^{2}(-z)-\frac{\pi^{2}}{6}
$$

for $z \notin(0,1)$, we obtain

$$
\begin{aligned}
m_{2}(x+y+1)= & \frac{1}{2 \pi i} \int_{|x|=1,|x+1| \leq 1} \frac{1}{2} \operatorname{Li}_{2}\left(|1+x|^{2}\right) \frac{d x}{x} \\
& +\frac{1}{2 \pi i} \int_{|x|=1,|x+1| \geq 1}\left(-\frac{1}{2} \Re \operatorname{Li}_{2}\left(|1+x|^{2}\right)+\frac{\pi^{2}}{6}\right) \frac{d x}{x} \\
= & \frac{1}{2 \pi i} \int_{|x|=1,|x+1| \leq 1} \operatorname{Li}_{2}\left(|1+x|^{2}\right) \frac{d x}{x}+\frac{\pi^{2}}{9} \\
= & \frac{1}{2 \pi} \int_{2 \pi / 3}^{4 \pi / 3} \operatorname{Li}_{2}\left(4 \cos ^{2}\left(\frac{\theta}{2}\right)\right) d \theta+\frac{\pi^{2}}{9} .
\end{aligned}
$$

Notice that

$$
\begin{aligned}
\int \cos ^{2 n} \theta d \theta= & \frac{\tan \theta}{2}\left(\begin{array}{c}
2 n-1 \\
n-1
\end{array}\right) \sum_{l=1}^{n} \frac{1}{2^{2 n-2 l}(2 l-1)\left(\begin{array}{c}
2 l-2 \\
l-1
\end{array}\right)} \cos ^{2 l} \theta \\
& +\frac{1}{2^{2 n-1}}\left(\begin{array}{c}
2 n-1 \\
n-1
\end{array}\right) \theta
\end{aligned}
$$

In particular,

$$
\int_{\pi / 3}^{2 \pi / 3} \cos ^{2 n} \theta d \theta=-\frac{\sqrt{3}}{2^{2 n}}\left(\begin{array}{c}
2 n-1 \\
n-1
\end{array}\right) \sum_{l=0}^{n-1} \frac{1}{(2 l+1)\left(\begin{array}{c}
2 l \\
l
\end{array}\right)}+\frac{1}{2^{2 n-1}}\left(\begin{array}{c}
2 n-1 \\
n-1
\end{array}\right) \frac{\pi}{3} .
$$

Now we use the identity for the sum of the inverses of Catalan numbers,

$$
\frac{2 \pi \sqrt{3}}{9}=\sum_{l=0}^{\infty} \frac{1}{(2 l+1)\left(\begin{array}{c}
2 l \\
l
\end{array}\right)}
$$


in order to get

$$
\int_{\pi / 3}^{2 \pi / 3} \cos ^{2 n} \theta d \theta=\frac{\sqrt{3}}{2^{2 n}}\left(\begin{array}{c}
2 n-1 \\
n-1
\end{array}\right) \sum_{l=n}^{\infty} \frac{1}{(2 l+1)\left(\begin{array}{c}
2 l \\
l
\end{array}\right)}
$$

Note that

$$
\frac{l ! l !}{(2 l+1) !}=B(l+1, l+1)=\int_{0}^{1} s^{l}(1-s)^{l} d s .
$$

Thus the above sum may be written as

$$
\sum_{l=n}^{\infty} \int_{0}^{1} s^{l}(1-s)^{l} d s=\int_{0}^{1} \frac{s^{n}(1-s)^{n}}{1-s(1-s)} d s
$$

Putting everything together yields

$$
\begin{aligned}
\frac{1}{2 \pi} \int_{2 \pi / 3}^{4 \pi / 3} \operatorname{Li}_{2}\left(4 \cos ^{2}\left(\frac{\theta}{2}\right)\right) d \theta+\frac{\pi^{2}}{9} & \\
& =\frac{\sqrt{3}}{\pi} \sum_{n=1}^{\infty} \frac{1}{n^{2}}\left(\begin{array}{c}
2 n-1 \\
n-1
\end{array}\right) \int_{0}^{1} \frac{s^{n}(1-s)^{n}}{1-s(1-s)} d s+\frac{\pi^{2}}{9} \\
& =\frac{\sqrt{3}}{2 \pi} \int_{0}^{1} \sum_{n=1}^{\infty} \frac{1}{n^{2}}\left(\begin{array}{c}
2 n \\
n
\end{array}\right) \frac{s^{n}(1-s)^{n}}{1-s(1-s)} d s+\frac{\pi^{2}}{9}
\end{aligned}
$$

At this point, we need the following

Lemma 12. For $|t| \leq 1 / 4$, we have

$$
\sum_{k=1}^{\infty}\left(\begin{array}{c}
2 k \\
k
\end{array}\right) \frac{t^{k}}{k^{2}}=2 \operatorname{Li}_{2}\left(\frac{1-\sqrt{1-4 t}}{2}\right)-\left(\log \left(\frac{1+\sqrt{1-4 t}}{2}\right)\right)^{2} .
$$

Proof. We start from the series

$$
\sum_{k=1}^{\infty}\left(\begin{array}{c}
2 k \\
k
\end{array}\right) t^{k}=-1+\sum_{k=0}^{\infty}\left(\begin{array}{c}
-1 / 2 \\
k
\end{array}\right)(-4 t)^{k}=-1+\frac{1}{\sqrt{1-4 t}}
$$

convergent for $|t| \leq 1 / 4$. By integration, we have

$$
\sum_{k=1}^{\infty}\left(\begin{array}{c}
2 k \\
k
\end{array}\right) \frac{t^{k}}{k}=-2 \log (1+\sqrt{1-4 t})+2 \log 2
$$

By integration again, we obtain the result. 
Now, if we set $t=s(1-s)$, we obtain $(1-\sqrt{1-4 t}) / 2=s$. Then the quantity (3) becomes

$$
\begin{aligned}
\frac{\sqrt{3}}{2 \pi} \int_{0}^{1}\left(2 \operatorname{Li}_{2}(s)-\log ^{2}(1-s)\right. & \frac{d s}{1-s(1-s)}+\frac{\pi^{2}}{9} \\
= & -\frac{\sqrt{3}}{\pi} \int_{0 \leq s_{1} \leq s_{2} \leq s \leq 1} \frac{d s_{1}}{s_{1}-1} \frac{d s_{2}}{s_{2}} \frac{d s}{1-s+s^{2}} \\
& -\frac{\sqrt{3}}{\pi} \int_{0 \leq s_{1} \leq s_{2} \leq s \leq 1} \frac{d s_{1}}{s_{1}-1} \frac{d s_{2}}{s_{2}-1} \frac{d s}{1-s+s^{2}}+\frac{\pi^{2}}{9} .
\end{aligned}
$$

But

$$
\frac{1}{1-s+s^{2}}=\frac{1}{i \sqrt{3}}\left(\frac{1}{s-\omega}-\frac{1}{s-\bar{\omega}}\right)
$$

where $\omega=(1+i \sqrt{3}) / 2$. Thus, the above equals

$$
\begin{aligned}
\frac{i}{\pi} \int_{0 \leq s_{1} \leq s_{2} \leq s \leq 1} \frac{d s_{1}}{s_{1}-1} \frac{d s_{2}}{s_{2}}\left(\frac{1}{s-\omega}-\frac{1}{s-\bar{\omega}}\right) d s \\
+\frac{i}{\pi} \int_{0 \leq s_{1} \leq s_{2} \leq s \leq 1} \frac{d s_{1}}{s_{1}-1} \frac{d s_{2}}{s_{2}-1}\left(\frac{1}{s-\omega}-\frac{1}{s-\bar{\omega}}\right) d s+\frac{\pi^{2}}{9} \\
=\frac{i}{\pi}\left(\operatorname{Li}_{2,1}(\omega, \bar{\omega})-\operatorname{Li}_{2,1}(\bar{\omega}, \omega)-\operatorname{Li}_{1,1,1}(1, \omega, \bar{\omega})\right. \\
\left.+\operatorname{Li}_{1,1,1}(1, \bar{\omega}, \omega)\right)+\frac{\pi^{2}}{9}
\end{aligned}
$$

where we have written the result in terms of polylogarithms.

Now

$$
\begin{aligned}
\operatorname{Li}_{1,1,1}(1, \bar{\omega}, \omega)-\operatorname{Li}_{1,1,1}(1, \omega, \bar{\omega}) & =\frac{5 i \pi^{3}}{81} \\
\operatorname{Li}_{2,1}(\bar{\omega}, \omega)-\operatorname{Li}_{2,1}(\omega, \bar{\omega}) & =\frac{7 i \pi^{3}}{162}
\end{aligned}
$$

(see for example [2]), and note that

$$
\frac{7 \pi^{2}}{162}-\frac{5 \pi^{2}}{81}+\frac{\pi^{2}}{9}=\frac{5 \pi^{2}}{54}
$$

The result should be compared to Smyth's formula

$$
m(x+y+1)=\frac{3 \sqrt{3}}{4 \pi} L\left(\chi_{-3}, 2\right)=L^{\prime}\left(\chi_{-3},-1\right) .
$$


3.2. $m_{2}(1+x+y(1-x))$

THEOREM 13.

$$
\begin{aligned}
m_{2}(1+ & x+y(1-x)) \\
= & \frac{4 i}{\pi}\left(\operatorname{Li}_{2,1}(-i,-i)-\operatorname{Li}_{2,1}(i, i)\right)+\frac{6 i}{\pi}\left(-\operatorname{Li}_{2,1}(-i, i)+\operatorname{Li}_{2,1}(i,-i)\right) \\
& +\frac{i}{\pi}\left(-\operatorname{Li}_{2,1}(1, i)+\operatorname{Li}_{2,1}(1,-i)\right)-\frac{7 \zeta(2)}{16}+\frac{\log 2}{\pi} L\left(\chi_{-4}, 2\right) .
\end{aligned}
$$

Proof. In order to apply the formula from Remark 9 (for the variable $y$ ) we need to have a rational function that is monic in $y$. Therefore, we divide by the factor $1+x$ :

$$
\begin{aligned}
& m_{2}(1-x+y(1+x)) \\
& \quad=m_{2}\left(\left(\frac{1-x}{1+x}\right)+y\right)+2 m\left(\left(\frac{1-x}{1+x}\right)+y, 1+x\right)+m_{2}(1+x) .
\end{aligned}
$$

For the first term, we have

$$
m_{2}\left(\left(\frac{1-x}{1+x}\right)+y\right)=\frac{1}{(2 \pi i)^{2}} \int_{|y|=1} \int_{|x|=1} \log ^{2}\left|\left(\frac{1-x}{1+x}\right)+y\right| \frac{d x}{x} \frac{d y}{y} .
$$

By applying Remark 9, this equals

$$
\begin{aligned}
\frac{1}{2 \pi i} \int_{|x|=1,|1-x| \leq|1+x|} \frac{1}{2} \operatorname{Li}_{2}\left(\left|\frac{1-x}{1+x}\right|^{2}\right) \frac{d x}{x} \\
+\frac{1}{2 \pi i} \int_{|x|=1,|1-x| \geq|1+x|} \frac{1}{2} \operatorname{Li}_{2}\left(\left|\frac{1+x}{1-x}\right|^{2}\right) \frac{d x}{x} \\
+\frac{1}{2 \pi i} \int_{|x|=1,|1-x| \geq|1+x|} \log ^{2}\left|\frac{1-x}{1+x}\right| \frac{d x}{x} \\
=\frac{1}{2 \pi i} \int_{|x|=1,|1-x| \leq|1+x|} \operatorname{Li}_{2}\left(\left|\frac{1-x}{1+x}\right|^{2}\right) \frac{d x}{x} \\
+\frac{1}{2 \pi i} \int_{|x|=1,|1-x| \geq|1+x|} \log ^{2}\left|\frac{1-x}{1+x}\right| \frac{d x}{x} .
\end{aligned}
$$

For the second term in (5) we obtain

$$
\begin{aligned}
m\left(\left(\frac{1-x}{1+x}\right)+y, 1+x\right) & \\
& =\frac{1}{(2 \pi i)^{2}} \int_{|y|=1} \int_{|x|=1} \log \left|\left(\frac{1-x}{1+x}\right)+y\right| \log |1+x| \frac{d x}{x} \frac{d y}{y} .
\end{aligned}
$$


By Jensen's formula with respect to the variable $y$, this equals

$$
\begin{aligned}
\frac{1}{2 \pi i} \int_{|x|=1} \log ^{+}\left|\frac{1-x}{1+x}\right| & \log |1+x| \frac{d x}{x} \\
& =\frac{1}{2 \pi i} \int_{|x|=1,|1-x| \geq|1+x|} \log \left|\frac{1-x}{1+x}\right| \log |1+x| \frac{d x}{x} .
\end{aligned}
$$

Then (5) becomes

$$
\text { (6) } \begin{aligned}
m_{2}(1- & x+y(1+x))=\frac{1}{2 \pi i} \int_{|x|=1,|1-x| \leq|1+x|} \operatorname{Li}_{2}\left(\left|\frac{1-x}{1+x}\right|^{2}\right) \frac{d x}{x} \\
& +\frac{1}{2 \pi i} \int_{|x|=1,|1-x| \geq|1+x|}\left(\log ^{2}|1-x|-\log ^{2}|1+x|\right) \frac{d x}{x}+\frac{\zeta(2)}{2} .
\end{aligned}
$$

For the first term on the right-hand side,

$$
\begin{aligned}
\frac{1}{2 \pi i} \int_{|x|=1,|1-x| \leq|1+x|} \operatorname{Li}_{2}\left(\left|\frac{1-x}{1+x}\right|^{2}\right) \frac{d x}{x} \\
=\frac{2}{\pi} \int_{-\pi / 4}^{\pi / 4} \operatorname{Li}_{2}\left(\tan ^{2} \theta\right) d \theta=\frac{4}{\pi} \int_{-\pi / 4}^{\pi / 4}\left(\operatorname{Li}_{2}(\tan \theta)+\operatorname{Li}_{2}(-\tan \theta)\right) d \theta .
\end{aligned}
$$

After the change of variables $y=\tan \theta$, this becomes

$$
\begin{aligned}
\frac{8}{\pi} \int_{0}^{1}\left(\operatorname{Li}_{2}(y)+\right. & \left.\operatorname{Li}_{2}(-y)\right) \frac{d y}{y^{2}+1} \\
& =\frac{4}{\pi} \int_{0}^{1}\left(\operatorname{Li}_{2}(y)+\operatorname{Li}_{2}(-y)\right)\left(\frac{1}{1+i y}+\frac{1}{1-i y}\right) d y \\
& =\frac{4}{\pi}\left(i \operatorname{Li}_{2,1}(i,-i)+i \operatorname{Li}_{2,1}(-i,-i)-i \operatorname{Li}_{2,1}(-i, i)-i \operatorname{Li}_{2,1}(i, i)\right) .
\end{aligned}
$$

For the second term in (6), we have

$$
\begin{aligned}
\frac{1}{2 \pi i} & \int_{|x|=1,|1-x| \geq|1+x|}\left(\log ^{2}|1-x|-\log ^{2}|1+x|\right) \frac{d x}{x} \\
= & \sum_{k, l \geq 1} \frac{1-(-1)^{k+l}}{k l} 2 \int_{1 / 4}^{3 / 4} \cos (2 \pi k \theta) \cos (2 \pi l \theta) d \theta \\
= & \sum_{k, l \geq 1} \frac{1-(-1)^{k+l}}{2 \pi k l}\left(\frac{i^{k+l+1}\left(1-(-1)^{k+l}\right)}{k+l}+\frac{i^{k-l+1}\left(1-(-1)^{k-l}\right)}{k-l}\right)
\end{aligned}
$$




$$
\begin{aligned}
= & \frac{i}{\pi} \sum_{k, l \geq 1} \frac{\left(1-(-1)^{k+l}\right) i^{k+l}}{k l^{2}}-\frac{i}{\pi} \sum_{k, l \geq 1} \frac{\left(1-(-1)^{k+l}\right) i^{k+l}}{(k+l) l^{2}} \\
& +\frac{2 i}{\pi} \sum_{k>l \geq 1} \frac{\left(1-(-1)^{k+l}\right) i^{k-l}}{(k-l) l^{2}}-\frac{2 i}{\pi} \sum_{k>l \geq 1} \frac{\left(1-(-1)^{k+l}\right) i^{k-l}}{k l^{2}} \\
= & \frac{i}{\pi}\left(\operatorname{Li}_{1}(i) \operatorname{Li}_{2}(i)-\operatorname{Li}_{1}(-i) \operatorname{Li}_{2}(-i)-\operatorname{Li}_{2,1}(1, i)+\operatorname{Li}_{2,1}(1,-i)\right) \\
& +\frac{2 i}{\pi}\left(\zeta(2)\left(\operatorname{Li}_{1}(i)-\operatorname{Li}_{1}(-i)\right)-\operatorname{Li}_{2,1}(-i, i)+\operatorname{Li}_{2,1}(i,-i)\right) \\
= & \frac{i}{\pi}\left(-i \log 2 L(\chi-4,2)-\frac{\pi i}{16} \zeta(2)-\operatorname{Li}_{2,1}(1, i)+\operatorname{Li}_{2,1}(1,-i)\right) \\
& +\frac{2 i}{\pi}\left(\zeta(2) \frac{\pi i}{2}-\operatorname{Li}_{2,1}(-i, i)+\operatorname{Li}_{2,1}(i,-i)\right)
\end{aligned}
$$

Putting everything together in (6), we obtain the final result

$$
\begin{aligned}
m_{2}(1-x+ & y(1+x)) \\
= & \frac{4 i}{\pi}\left(\operatorname{Li}_{2,1}(-i,-i)-\operatorname{Li}_{2,1}(i, i)\right)+\frac{6 i}{\pi}\left(-\operatorname{Li}_{2,1}(-i, i)+\operatorname{Li}_{2,1}(i,-i)\right) \\
& +\frac{i}{\pi}\left(-\operatorname{Li}_{2,1}(1, i)+\operatorname{Li}_{2,1}(1,-i)\right)-\frac{7 \zeta(2)}{16}+\frac{\log 2}{\pi} L\left(\chi_{-4}, 2\right) .
\end{aligned}
$$

The previous result should be compared to (see [6])

$$
m(1-x+y(1+x))=\frac{2}{\pi} L\left(\chi_{-4}, 2\right) .
$$

4. Zeta Mahler measures. In this section, we consider zeta Mahler measures. We compute some examples and apply them to the computation of higher Mahler measures.

4.1. $Z(s, x-1)$. As usual, we start with the linear polynomial $x-1$.

THEOREM 14.

$$
Z(s, x-1)=\int_{0}^{1}(2 \sin \pi \theta)^{s} d \theta=\exp \left(\sum_{k=2}^{\infty} \frac{(-1)^{k}\left(1-2^{1-k}\right) \zeta(k)}{k} s^{k}\right)
$$

around $s=0$.

This result is a particular case of a formula obtained by Akatsuka [1]. 
Proof. First we show that

$$
Z(s, x-1)=\frac{\Gamma(s+1)}{\Gamma(s / 2+1)^{2}}=\frac{s !}{((s / 2) !)^{2}}=\left(\begin{array}{c}
s \\
s / 2
\end{array}\right),
$$

where $s !=\Gamma(s+1)$. In fact,

$$
Z(s, x-1)=2^{s+1} \int_{0}^{1 / 2}(\sin \pi \theta)^{s} d \theta .
$$

After the change of variables $t=\sin ^{2} \pi \theta$ this becomes

$$
\frac{2^{s}}{\pi} \int_{0}^{1} t^{(s-1) / 2}(1-t)^{-1 / 2} d t,
$$

so we have obtained the beta function:

$$
Z(s, x-1)=\frac{2^{s}}{\pi} B\left(\frac{s+1}{2}, \frac{1}{2}\right)=\frac{2^{s}}{\pi} \frac{\Gamma\left(\frac{s+1}{2}\right) \Gamma\left(\frac{1}{2}\right)}{\Gamma\left(\frac{s}{2}+1\right)}=\frac{2^{s}}{\sqrt{\pi}} \frac{\Gamma\left(\frac{s+1}{2}\right)}{\Gamma\left(\frac{s}{2}+1\right)} .
$$

Hence, by using

$$
\Gamma\left(\frac{s+1}{2}\right)=\frac{\Gamma(s)}{\Gamma\left(\frac{s}{2}\right)} 2^{1-s} \pi^{1 / 2}=\frac{\Gamma(s+1)}{\Gamma\left(\frac{s}{2}+1\right)} 2^{-s} \pi^{1 / 2},
$$

we conclude that

$$
Z(s, x-1)=\frac{\Gamma(s+1)}{\Gamma\left(\frac{s}{2}+1\right)^{2}} .
$$

On the other hand, the product expression

$$
\Gamma(s+1)^{-1}=e^{\gamma s} \prod_{n=1}^{\infty}\left(1+\frac{s}{n}\right) e^{-s / n}
$$

yields

$$
\begin{aligned}
Z(s, x-1) & =\prod_{n=1}^{\infty} \frac{(1+s / 2 n)^{2}}{1+s / n}=\exp \left(\sum_{n=1}^{\infty}\left\{2 \log \left(1+\frac{s}{2 n}\right)-\log \left(1+\frac{s}{n}\right)\right\}\right) \\
& =\exp \left(\sum_{k=1}^{\infty} \frac{(-1)^{k-1}}{k} \sum_{n=1}^{\infty}\left\{2\left(\frac{1}{2 n}\right)^{k}-\frac{1}{n^{k}}\right\} s^{k}\right) \\
& =\exp \left(\sum_{k=2}^{\infty} \frac{(-1)^{k-1}}{k} \zeta(k)\left(2^{1-k}-1\right) s^{k}\right) \\
& =\exp \left(\sum_{k=2}^{\infty} \frac{(-1)^{k}\left(1-2^{1-k}\right) \zeta(k)}{k} s^{k}\right) .
\end{aligned}
$$

An analogous idea for evaluating $Z(s, P)$ appears in [5]. 
4.2. $m_{k}(x-1)$. We can now use the evaluation of $Z(s, x-1)$ to recover the formula for $m_{k}(x-1)$. From Theorem 14,

$$
\begin{aligned}
Z(s, x-1)= & \exp \left(\frac{\zeta(2)}{4} s^{2}-\frac{\zeta(3)}{4} s^{3}+\frac{7 \zeta(4)}{32} s^{4}+\cdots\right) \\
= & 1+\frac{\zeta(2)}{4} s^{2}-\frac{\zeta(3)}{4} s^{3} \\
& +\left(\frac{7 \zeta(4)}{32}+\frac{\zeta(2)^{2}}{32}\right) s^{4}+\cdots .
\end{aligned}
$$

On the other hand, by construction,

$$
\begin{aligned}
Z(s, x-1)=1+m_{1}(x-1) & s+\frac{1}{2} m_{2}(x-1) s^{2} \\
+ & \frac{1}{6} m_{3}(x-1) s^{3}+\frac{1}{24} m_{4}(x-1) s^{4}+\cdots .
\end{aligned}
$$

Putting both identities together, we recover the result from Theorem 3. In particular,

$$
\begin{aligned}
& m_{1}(x-1)=0 \\
& m_{2}(x-1)=\frac{\zeta(2)}{2}=\frac{\pi^{2}}{12} \\
& m_{3}(x-1)=-\frac{3 \zeta(3)}{2} \\
& m_{4}(x-1)=\frac{3}{4}\left(7 \zeta(4)+\zeta(2)^{2}\right)=\frac{19 \pi^{4}}{240}, \quad \cdots
\end{aligned}
$$

5. A computation of higher zeta Mahler measure. We compute the simplest example of a higher zeta Mahler measure and apply it to multiple higher Mahler measures.

THEOREM 15.

$$
\begin{aligned}
Z(s, t ; x-1, x+1) & =\int_{0}^{1}|2 \sin \pi \theta|^{s}|2 \cos \pi \theta|^{t} d \theta \\
& =\frac{\Gamma(s+1) \Gamma(t+1)}{\Gamma\left(\frac{s}{2}+1\right) \Gamma\left(\frac{t}{2}+1\right) \Gamma\left(\frac{s+t}{2}+1\right)} \\
& =\frac{s ! t !}{\left(\frac{s}{2}\right) !\left(\frac{t}{2}\right) !\left(\frac{s+t}{2}\right) !} \\
& =\prod_{n=1}^{\infty} \frac{\left(1+\frac{s}{2 n}\right)\left(1+\frac{t}{2 n}\right)\left(1+\frac{s+t}{2 n}\right)}{\left(1+\frac{s}{n}\right)\left(1+\frac{t}{n}\right)} .
\end{aligned}
$$


(ii) $\quad Z(s, t ; x-1, x+1)$

$$
\begin{aligned}
& =\exp \left(\sum_{k=2}^{\infty} \frac{(-1)^{k}}{k} \zeta(k)\left\{\left(1-2^{-k}\right)\left(s^{k}+t^{k}\right)-2^{-k}(s+t)^{k}\right\}\right) \\
& \in \mathbb{Q}\left[\pi^{2}, \zeta(3), \zeta(5), \ldots\right][[s, t]]
\end{aligned}
$$

around $s=t=0$.

(iii)

$$
\begin{aligned}
& m(\underbrace{x-1, \ldots, x-1}_{k}, \underbrace{x+1, \ldots, x+1}_{l}) \\
& =\int_{0}^{1}(\log |2 \sin \pi \theta|)^{k}(\log |2 \cos \pi \theta|)^{l} d \theta
\end{aligned}
$$

belongs to $\mathbb{Q}\left[\pi^{2}, \zeta(3), \zeta(5), \zeta(7), \ldots\right]$ for integers $k, l \geq 0$.

Proof. (i) By definition,

$$
\begin{aligned}
Z(s, t ; x-1, x+1) & =2^{s+t} \int_{0}^{1}(\sin \pi \theta)^{s}|\cos \pi \theta|^{t} d \theta \\
& =2^{s+t+1} \int_{0}^{1 / 2}(\sin \pi \theta)^{s}(\cos \pi \theta)^{t} d \theta .
\end{aligned}
$$

By the change of variables $u=\sin ^{2} \pi \theta$,

$$
\begin{aligned}
Z(s, t ; x-1, x+1) & =\frac{2^{s+t}}{\pi} \int_{0}^{1} u^{(s-1) / 2}(1-u)^{(t-1) / 2} d u \\
& =\frac{2^{s+t}}{\pi} B\left(\frac{s+1}{2}, \frac{t+1}{2}\right) \\
& =\frac{2^{s+t}}{\pi} \frac{\Gamma\left(\frac{s+1}{2}\right) \Gamma\left(\frac{t+1}{2}\right)}{\Gamma\left(\frac{s+t}{2}+1\right)} .
\end{aligned}
$$

We now use again the identity

$$
\Gamma\left(\frac{z+1}{2}\right)=2^{-z} \pi^{1 / 2} \frac{\Gamma(z+1)}{\Gamma\left(\frac{z}{2}+1\right)},
$$

to get

$$
\begin{aligned}
Z(s, t ; x-1, x+1) & =\frac{\Gamma(s+1) \Gamma(t+1)}{\Gamma\left(\frac{s}{2}+1\right) \Gamma\left(\frac{t}{2}+1\right) \Gamma\left(\frac{s+t}{2}+1\right)} \\
& =\prod_{n=1}^{\infty} \frac{\left(1+\frac{s}{2 n}\right)\left(1+\frac{t}{2 n}\right)\left(1+\frac{s+t}{2 n}\right)}{\left(1+\frac{s}{n}\right)\left(1+\frac{t}{n}\right)}
\end{aligned}
$$


(ii) The above expression yields

$$
\begin{aligned}
& Z(s, t ; x-1, x+1) \\
& =\exp \left(\sum _ { n = 1 } ^ { \infty } \left\{\log \left(1+\frac{s}{2 n}\right)+\log \left(1+\frac{t}{2 n}\right)+\log \left(1+\frac{s+t}{2 n}\right)\right.\right. \\
& \left.\left.\quad-\log \left(1+\frac{s}{n}\right)-\log \left(1+\frac{t}{n}\right)\right\}\right) \\
& =\exp \left(\sum_{k=1}^{\infty} \frac{(-1)^{k-1}}{k} \sum_{n=1}^{\infty}\left\{\left(\frac{s}{2 n}\right)^{k}+\left(\frac{t}{2 n}\right)^{k}+\left(\frac{s+t}{2 n}\right)^{k}-\left(\frac{s}{n}\right)^{k}-\left(\frac{t}{n}\right)^{k}\right\}\right) \\
& =\exp \left(\sum_{k=2}^{\infty} \frac{(-1)^{k-1}}{k} \zeta(k)\left\{2^{-k} s^{k}+2^{-k} t^{k}+2^{-k}(s+t)^{k}-s^{k}-t^{k}\right\}\right) \\
& =\exp \left(\sum_{k=2}^{\infty} \frac{(-1)^{k}}{k} \zeta(k)\left\{\left(1-2^{-k}\right) s^{k}+\left(1-2^{-k}\right) t^{k}-2^{-k}(s+t)^{k}\right\}\right) .
\end{aligned}
$$

This power series belongs to $\mathbb{Q}\left[\pi^{2}, \zeta(3), \zeta(5), \zeta(7), \ldots\right][[s, t]]$.

(iii) From (ii), we see that

$$
\frac{\partial^{k+l}}{\partial s^{k} \partial t^{l}} Z(0,0 ; x-1, x+1) \in \mathbb{Q}\left[\pi^{2}, \zeta(3), \zeta(5), \zeta(7), \ldots\right],
$$

which is simply

$$
\begin{aligned}
& m(\underbrace{x-1, \ldots, x-1}_{k}, \underbrace{x+1, \ldots,}_{l}x+1) \\
&=\int_{0}^{1}(\log |2 \sin \pi \theta|)^{k}(\log |2 \cos \pi \theta|)^{l} d \theta .
\end{aligned}
$$

EXAMPLE 16. In order to compute examples, we compare the terms of lowest degrees in the two expressions of $Z(s, t ; x-1, x+1)$. On the one hand, we have

$$
\begin{aligned}
Z(s, t & ; x-1, x+1) \\
= & \exp \left(\frac{\zeta(2)}{2}\left(\frac{3}{4}\left(s^{2}+t^{2}\right)-\frac{1}{4}(s+t)^{2}\right)\right. \\
& \left.\quad-\frac{\zeta(3)}{3}\left(\frac{7}{8}\left(s^{3}+t^{3}\right)-\frac{1}{8}(s+t)^{3}\right)+(\text { degree } \geq 4)\right) \\
= & \exp \left(\frac{\zeta(2)}{4}\left(s^{2}+t^{2}-s t\right)-\frac{\zeta(3)}{8}\left(2 s^{3}+2 t^{3}-s^{2} t-s t^{2}\right)+(\text { degree } \geq 4)\right) .
\end{aligned}
$$


On the other hand,

$$
\begin{aligned}
Z(s, & ; x-1, x+1) \\
= & +\left(\frac{1}{2} m(x-1, x-1) s^{2}+\frac{1}{2} m(x+1, x+1) t^{2}+m(x+1, x-1) s t\right) \\
& +\left(\frac{1}{6} m(x-1, x-1, x-1) s^{3}+\frac{1}{6} m(x+1, x+1, x+1) t^{3}\right. \\
& \left.+\frac{1}{2} m(x-1, x-1, x+1) s^{2} t+\frac{1}{2} m(x-1, x+1, x+1) s t^{2}\right) \\
& +(\text { degree } \geq 4) .
\end{aligned}
$$

We obtain:

$$
\begin{aligned}
m(x-1, x+1) & =\int_{0}^{1} \log |2 \sin \pi \theta| \log |2 \cos \pi \theta| d \theta=-\frac{\zeta(2)}{4}=-\frac{\pi^{2}}{24}, \\
m(x-1, x-1, x+1) & =\int_{0}^{1}(\log |2 \sin \pi \theta|)^{2} \log |2 \cos \pi \theta| d \theta=2 \frac{\zeta(3)}{8}=\frac{\zeta(3)}{4}, \\
m(x-1, x+1, x+1) & =\int_{0}^{1} \log |2 \sin \pi \theta|(\log |2 \cos \pi \theta|)^{2} d \theta=2 \frac{\zeta(3)}{8}=\frac{\zeta(3)}{4} .
\end{aligned}
$$

Note that the calculation

$$
Z(s, 0 ; x-1, x+1)=Z(s, x-1)=\left(\begin{array}{c}
s \\
s / 2
\end{array}\right)
$$

yields $m_{k}(x-1)$ again.

We also remark that we have another relation:

$$
Z(s, s ; x-1, x+1)=Z(s, x-1)=Z(s, x+1) .
$$

\section{Further examples}

6.1. The case $P=x+x^{-1}+y+y^{-1}+c$

Theorem 17. For $c>4$,

$$
\begin{aligned}
Z\left(s, x+x^{-1}+y+y^{-1}+c\right) & =c^{s} \sum_{j=0}^{\infty}\left(\begin{array}{c}
s \\
2 j
\end{array}\right) \frac{1}{c^{2 j}}\left(\begin{array}{c}
2 j \\
j
\end{array}\right)^{2} \\
& =c^{s}{ }_{3} F_{2}\left(\begin{array}{c}
-\frac{s}{2}, \frac{1-s}{2}, \frac{1}{2} \\
1,1
\end{array} \mid \frac{16}{c^{2}}\right),
\end{aligned}
$$

where the generalized hypergeometric series ${ }_{3} F_{2}$ is defined by

$$
{ }_{3} F_{2}\left(\begin{array}{c}
a_{1}, a_{2}, a_{3} \\
b_{1}, b_{2}
\end{array} \mid z\right)=\sum_{j=0}^{\infty} \frac{\left(a_{1}\right)_{j}\left(a_{2}\right)_{j}\left(a_{3}\right)_{j}}{\left(b_{1}\right)_{j}\left(b_{2}\right)_{j} j !} z^{j},
$$

with the Pochhammer symbol defined by $(a)_{j}=a(a+1) \cdots(a+j-1)$. 
Proof. We first write

$$
x+x^{-1}+y+y^{-1}+c=c\left(\frac{x+x^{-1}+y+y^{-1}}{c}+1\right) .
$$

Since $c \geq 4,\left(x+x^{-1}+y+y^{-1}\right) / c+1$ is a positive number in the unit torus. Hence, we may omit the absolute value in the computation of the zeta function. Therefore we may write

$$
\begin{aligned}
Z\left(s, x+x^{-1}\right. & \left.+y+y^{-1}+c\right) \\
& =\frac{1}{(2 \pi i)^{2}} \int_{|y|=1} \int_{|x|=1}\left(x+x^{-1}+y+y^{-1}+c\right)^{s} \frac{d x}{x} \frac{d y}{y} \\
& =\frac{c^{s}}{(2 \pi i)^{2}} \int_{|y|=1} \int_{|x|=1}\left(1+\frac{x+x^{-1}+y+y^{-1}}{c}\right)^{s} \frac{d x}{x} \frac{d y}{y} \\
& =c^{s} \sum_{k=0}^{\infty}\left(\begin{array}{c}
s \\
k
\end{array}\right) \frac{1}{(2 \pi i)^{2}} \int_{|y|=1} \int_{|x|=1}\left(\frac{x+x^{-1}+y+y^{-1}}{c}\right)^{k} \frac{d x}{x} \frac{d y}{y} \\
& =c^{s} \sum_{j=0}^{\infty}\left(\begin{array}{c}
s \\
2 j
\end{array}\right) \frac{1}{c^{2 j}}\left(\begin{array}{c}
2 j \\
j
\end{array}\right)^{2} .
\end{aligned}
$$

The last equality is the result of the following observation. The number

$$
\frac{1}{(2 \pi i)^{2}} \int_{|y|=1} \int_{|x|=1}\left(x+x^{-1}+y+y^{-1}\right)^{k} \frac{d x}{x} \frac{d y}{y}
$$

is the constant coefficient of $\left(x+x^{-1}+y+y^{-1}\right)^{k}$. This was observed by Rodríguez-Villegas [4] who studied this specific example as part of the computation of the classical Mahler measure for this family of polynomials.

The expression in terms of the generalized hypergeometric function is derived from $\left(\begin{array}{c}s \\ 2 j\end{array}\right)(2 j) !=2^{2 j}\left(-\frac{s}{2}\right)_{j}\left(\frac{1-s}{2}\right)_{j}$ and $(2 j) !=2^{2 j}\left(\frac{1}{2}\right)_{j} j !$. Note that the series ${ }_{3} F_{2}(z)$ converges in $|z|<1$, which is compatible with the condition $c>4$ in the statement of the theorem.

6.2. Properties of zeta Mahler measures. The proof of Theorem 17 may also be achieved by combining the following elementary properties of zeta Mahler measures:

LEMMA 18.

(i) For a positive constant $\lambda$, we have $Z(s, \lambda P)=\lambda^{s} Z(s, P)$.

(ii) Let $P \in \mathbb{C}\left[x_{1}^{ \pm 1}, \ldots, x_{n}^{ \pm 1}\right]$ be a Laurent polynomial that takes nonnegative real values in the unit torus. Then we have the following series expansion for $|\lambda| \leq 1 / \max (P)$, where $\max (P)$ is the maxi- 
mum of $P$ on the unit torus:

$$
\begin{aligned}
Z(s, 1+\lambda P) & =\sum_{k=0}^{\infty}\left(\begin{array}{l}
s \\
k
\end{array}\right) Z(k, P) \lambda^{k}, \\
m(1+\lambda P) & =\sum_{k=1}^{\infty} \frac{(-1)^{k-1}}{k} Z(k, P) \lambda^{k} .
\end{aligned}
$$

More generally,

$$
m_{j}(1+\lambda P)=j ! \sum_{0<k_{1}<\cdots<k_{j}} \frac{(-1)^{k_{j}-j}}{k_{1} \cdots k_{j}} Z\left(k_{j}, P\right) \lambda^{k_{j}} .
$$

(iii) $Z(s, P)=Z(s / 2, P \bar{P})$, where we put $\bar{P}=\sum_{\alpha} \bar{a}_{\alpha} x^{-\alpha}$ for $P=$ $\sum_{\alpha} a_{\alpha} x^{\alpha}$. Note that $P \bar{P}$ is real-valued on the torus.

Therefore, in principle, the knowledge of $m(1+\lambda P)$ yields enough information to determine $Z(s, 1+\lambda P)$.

Proof. (i) and (iii) are obvious. For (ii), we may use the Taylor expansions in $\lambda$,

$$
(1+\lambda P)^{s}=\sum_{k=0}^{\infty}\left(\begin{array}{l}
s \\
k
\end{array}\right) \lambda^{k} P^{k}, \quad \log (1+\lambda P)=\sum_{k=1}^{\infty} \frac{(-1)^{k-1}}{k} \lambda^{k} P^{k} .
$$

In particular, we may write

$$
Z(s, 1+\lambda P)=\sum_{k=0}^{\infty} m_{k}(1+\lambda P) \frac{s^{k}}{k !}=\sum_{k=0}^{\infty} Z(k, P) \lambda^{k} \frac{s(s-1) \cdots(s-k+1)}{k !} .
$$

In other words, the coefficients with respect to the monomial basis are the $k$-logarithmic Mahler measures $m_{k}(1+\lambda P)$, while the coefficients with respect to the shifted monomial basis are the (special values of) zeta Mahler measures $Z(k, P) \lambda^{k}$.

Combining these observations, we obtain the three equalities.

6.3. The case $P=x+y+c$. Now we apply these ideas to $P=x+y+c$ with $c \geq 2$.

TheOREM 19. Let $c \geq 2$. Then

$$
\begin{aligned}
& Z(s, x+y+c)=c^{s} \sum_{j=0}^{\infty}\left(\begin{array}{c}
s / 2 \\
j
\end{array}\right)^{2} \frac{1}{c^{2 j}}\left(\begin{array}{c}
2 j \\
j
\end{array}\right), \\
& m_{2}(x+y+c)=\log ^{2} c+\frac{1}{2} \sum_{k=1}^{\infty}\left(\begin{array}{c}
2 k \\
k
\end{array}\right) \frac{1}{k^{2} c^{2 k}},
\end{aligned}
$$


(iii) $\quad m_{3}(x+y+c)$

$$
=\log ^{3} c+\frac{3}{2} \log c \sum_{k=1}^{\infty}\left(\begin{array}{c}
2 k \\
k
\end{array}\right) \frac{1}{k^{2} c^{2 k}}-\frac{3}{2} \sum_{k=2}^{\infty}\left(\begin{array}{c}
2 k \\
k
\end{array}\right) \frac{1}{k^{2} c^{2 k}} \sum_{j=1}^{k-1} \frac{1}{j} .
$$

In particular, we obtain the special values

$$
\begin{aligned}
& m_{2}(x+y+2)=\frac{\zeta(2)}{2} \\
& m_{3}(x+y+2)=\frac{9}{2} \log 2 \zeta(2)-\frac{15}{4} \zeta(3) .
\end{aligned}
$$

Proof. (i) In this case, the polynomial is not reciprocal, so we first need to consider $(x+y+c)\left(x^{-1}+y^{-1}+c\right)$. Then

$$
\begin{aligned}
Z(s, x+y+c)= & Z\left(s / 2,(x+y+c)\left(x^{-1}+y^{-1}+c\right)\right) \\
= & \frac{1}{(2 \pi i)^{2}} \int_{|y|=1} \int_{|x|=1}\left((x+y+c)\left(x^{-1}+y^{-1}+c\right)\right)^{s / 2} \frac{d x}{x} \frac{d y}{y} \\
= & \frac{c^{s}}{(2 \pi i)^{2}} \int_{|y|=1} \int_{|x|=1}\left(1+\frac{x+y}{c}\right)^{s / 2}\left(1+\frac{x^{-1}+y^{-1}}{c}\right)^{s / 2} \frac{d x}{x} \frac{d y}{y} \\
= & c^{s} \sum_{j=0}^{\infty} \sum_{k=0}^{\infty}\left(\begin{array}{c}
s / 2 \\
j
\end{array}\right)\left(\begin{array}{c}
s / 2 \\
k
\end{array}\right) \\
& \times \frac{1}{(2 \pi i)^{2}} \int_{|y|=1} \int_{|x|=1}\left(\frac{x+y}{c}\right)^{j}\left(\frac{x^{-1}+y^{-1}}{c}\right)^{k} \frac{d x}{x} \frac{d y}{y} \\
= & c^{s} \sum_{j=0}^{\infty}\left(\begin{array}{c}
s / 2 \\
j
\end{array}\right)^{2} \frac{1}{c^{2 j}}\left(\begin{array}{c}
2 j \\
j
\end{array}\right) .
\end{aligned}
$$

The last identity was obtained, as in the case of $x+x^{-1}+y+y^{-1}+c$, by computing the constant coefficient of the product of powers of polynomials in the integrand.

Formulas (ii) and (iii) are consequences of (i) and Lemma 18.

If we set $t=1 / 4$ in the equation of Lemma 12, we obtain $\zeta(2)-2 \log ^{2} 2$. Combining this with (ii), we get the result of (iv).

For the last formula (v), it is enough to prove the following identity:

$$
\sum_{k=2}^{\infty}\left(\begin{array}{c}
2 k \\
k
\end{array}\right) \frac{1}{k^{2} 4^{k}} \sum_{j=1}^{k-1} \frac{1}{j}=-\frac{4}{3} \log ^{3} 2-2 \zeta(2) \log 2+\frac{5}{2} \zeta(3) .
$$

We have

$$
\sum_{k=1}^{\infty}\left(\begin{array}{c}
2 k \\
k
\end{array}\right) \frac{t^{k}}{k^{2}}=2 \operatorname{Li}_{2}\left(\frac{1-\sqrt{1-4 t}}{2}\right)-\left(\log \left(\frac{1+\sqrt{1-4 t}}{2}\right)\right)^{2} .
$$


Now we turn the left-hand side into a double series:

$$
\begin{aligned}
\sum_{k=2}^{\infty}\left(\begin{array}{c}
2 k \\
k
\end{array}\right) & \frac{t^{k}}{k^{2}} \sum_{j=0}^{k-2} x^{j}=\sum_{k=2}^{\infty}\left(\begin{array}{c}
2 k \\
k
\end{array}\right) \frac{t^{k}}{k^{2}}\left(\frac{x^{k-1}-1}{x-1}\right) \\
= & \frac{1}{x(x-1)}\left(2 \operatorname{Li}_{2}\left(\frac{1-\sqrt{1-4 x t}}{2}\right)-\left(\log \left(\frac{1+\sqrt{1-4 x t}}{2}\right)\right)^{2}\right) \\
& -\frac{1}{x-1}\left(2 \operatorname{Li}_{2}\left(\frac{1-\sqrt{1-4 t}}{2}\right)-\left(\log \left(\frac{1+\sqrt{1-4 t}}{2}\right)\right)^{2}\right) .
\end{aligned}
$$

In particular, by evaluating at $t=1 / 4$, we obtain

$$
\begin{aligned}
\sum_{k=2}^{\infty}\left(\begin{array}{c}
2 k \\
k
\end{array}\right) \frac{1}{k^{2} 4^{k}} & \sum_{j=0}^{k-2} x^{j} \\
= & \frac{1}{x(x-1)}\left(2 \operatorname{Li}_{2}\left(\frac{1-\sqrt{1-x}}{2}\right)-\left(\log \left(\frac{1+\sqrt{1-x}}{2}\right)\right)^{2}\right) \\
& -\frac{1}{x-1}\left(\zeta(2)-2 \log ^{2} 2\right) .
\end{aligned}
$$

Integrating from 0 to 1 , we obtain the double series that we wish to evaluate:

$$
\begin{aligned}
& I:=\sum_{k=2}^{\infty}\left(\begin{array}{c}
2 k \\
k
\end{array}\right) \frac{1}{k^{2} 4^{k}} \sum_{j=1}^{k-1} \frac{1}{j} \\
&=\int_{0}^{1}\left(\frac { 1 } { x ( x - 1 ) } \left(2 \operatorname{Li}_{2}\left(\frac{1-\sqrt{1-x}}{2}\right)\right.\right.\left.-\left(\log \left(\frac{1+\sqrt{1-x}}{2}\right)\right)^{2}\right) \\
&\left.-\frac{1}{x-1}\left(\zeta(2)-2 \log ^{2} 2\right)\right) d x .
\end{aligned}
$$

We just need to perform the integration. For that, we consider the change of variables $y=(1-\sqrt{1-x}) / 2$ :

$$
\begin{aligned}
I= & \int_{0}^{1 / 2}\left(2 \operatorname{Li}_{2}(y)-(\log (1-y))^{2}\right)\left(\frac{4}{2 y-1}-\frac{1}{y-1}-\frac{1}{y}\right) d y \\
& -4\left(\zeta(2)-2 \log ^{2} 2\right) \int_{0}^{1 / 2} \frac{d y}{2 y-1} .
\end{aligned}
$$

We write the expression in terms of iterated integrals, so that we can relate the result to multiple polylogarithms:

$$
\begin{array}{r}
2 \operatorname{Li}_{2}(y)-(\log (1-y))^{2} \\
\quad=-2 \int_{0 \leq t_{1} \leq t_{2} \leq y} \frac{d t_{1}}{t_{1}-1} \frac{d t_{2}}{t_{2}}-2 \int_{0 \leq t_{1} \leq t_{2} \leq y} \frac{d t_{1}}{t_{1}-1} \frac{d t_{2}}{t_{2}-1} .
\end{array}
$$


We have

$$
\begin{aligned}
I= & -2 \int_{0 \leq t_{1} \leq t_{2} \leq y \leq 1 / 2} \frac{d t_{1}}{t_{1}-1} \frac{d t_{2}}{t_{2}}\left(\frac{4}{2 y-1}-\frac{1}{y-1}-\frac{1}{y}\right) d y \\
& -2 \int_{0 \leq t_{1} \leq t_{2} \leq y \leq 1 / 2} \frac{d t_{1}}{t_{1}-1} \frac{d t_{2}}{t_{2}-1}\left(\frac{4}{2 y-1}-\frac{1}{y-1}-\frac{1}{y}\right) d y \\
& +\left(2 \int_{0 \leq t_{1} \leq t_{2} \leq 1 / 2} \frac{d t_{1}}{t_{1}-1} \frac{d t_{2}}{t_{2}}+2 \int_{0 \leq t_{1} \leq t_{2} \leq 1 / 2} \frac{d t_{1}}{t_{1}-1} \frac{d t_{2}}{t_{2}-1}\right) \int_{0}^{1 / 2} \frac{4 d y}{2 y-1} .
\end{aligned}
$$

After some rearranging we get

$$
\begin{aligned}
I= & 2 \int_{0 \leq t_{1} \leq t_{2} \leq y \leq 1 / 2} \frac{d t_{1}}{t_{1}-1} \frac{d t_{2}}{t_{2}}\left(\frac{1}{y-1}+\frac{1}{y}\right) d y \\
& +2 \int_{0 \leq t_{1} \leq t_{2} \leq y \leq 1 / 2} \frac{d t_{1}}{t_{1}-1} \frac{d t_{2}}{t_{2}-1}\left(\frac{1}{y-1}+\frac{1}{y}\right) d y \\
& +8 \int_{0 \leq y, t_{1} \leq t_{2} \leq 1 / 2} \frac{d y}{2 y-1} \frac{d t_{1}}{t_{1}-1} \frac{d t_{2}}{t_{2}}+8 \int_{0 \leq y, t_{1} \leq t_{2} \leq 1 / 2} \frac{d y}{2 y-1} \frac{d t_{1}}{t_{1}-1} \frac{d t_{2}}{t_{2}-1} .
\end{aligned}
$$

We make the change of variables $s_{i}=2 t_{i}, z=2 y$. Then

$$
\begin{aligned}
I= & 2 \int_{0 \leq s_{1} \leq s_{2} \leq z \leq 1} \frac{d s_{1}}{s_{1}-2} \frac{d s_{2}}{s_{2}}\left(\frac{1}{z-2}+\frac{1}{z}\right) d z \\
& +2 \int_{0 \leq s_{1} \leq s_{2} \leq z \leq 1} \frac{d s_{1}}{s_{1}-2} \frac{d s_{2}}{s_{2}-2}\left(\frac{1}{z-2}+\frac{1}{z}\right) d z \\
& +4 \int_{0 \leq z, s_{1} \leq s_{2} \leq 1} \frac{d z}{z-1} \frac{d s_{1}}{s_{1}-2} \frac{d s_{2}}{s_{2}}+4 \int_{0 \leq z, s_{1} \leq s_{2} \leq 1} \frac{d z}{z-1} \frac{d s_{1}}{s_{1}-2} \frac{d s_{2}}{s_{2}-2} .
\end{aligned}
$$

Now we make another change of variables $u_{i}=1-s_{i}, w=1-z$ to get

$$
\begin{aligned}
I= & -2 \int_{0 \leq w \leq u_{2} \leq u_{1} \leq 1}\left(\frac{1}{w+1}+\frac{1}{w-1}\right) d w \frac{d u_{2}}{u_{2}-1} \frac{d u_{1}}{u_{1}+1} \\
& -2 \int_{0 \leq w \leq u_{2} \leq u_{1} \leq 1}\left(\frac{1}{w+1}+\frac{1}{w-1}\right) d w \frac{d u_{2}}{u_{2}+1} \frac{d u_{1}}{u_{1}+1} \\
& -4 \int_{0 \leq u_{2} \leq w, u_{1} \leq 1} \frac{d u_{2}}{u_{2}-1} \frac{d u_{1}}{u_{1}+1} \frac{d w}{w}-4 \int_{0 \leq u_{2} \leq w, u_{1} \leq 1} \frac{d u_{2}}{u_{2}+1} \frac{d u_{1}}{u_{1}+1} \frac{d w}{w} .
\end{aligned}
$$

We may now express all the terms as hyperlogarithms, and then as multiple 
polylogarithms evaluated at \pm 1 :

$$
\begin{aligned}
I= & -2 I_{1,1,1}(-1,1,-1,1)-2 I_{1,1,1}(1,1,-1,1) \\
& -2 I_{1,1,1}(-1,-1,-1,1)-2 I_{1,1,1}(1,-1,-1,1) \\
& -4 I_{1,2}(1,-1,1)-4 I_{2,1}(1,-1,1)-4 I_{1,2}(-1,-1,1)-4 I_{2,1}(-1,-1,1) \\
= & 2 \operatorname{Li}_{1,1,1}(-1,-1,-1)+2 \operatorname{Li}_{1,1,1}(1,-1,-1) \\
& +2 \operatorname{Li}_{1,1,1}(1,1,-1)+2 \operatorname{Li}_{1,1,1}(-1,1,-1) \\
& -4 \operatorname{Li}_{1,2}(-1,-1)-4 \operatorname{Li}_{2,1}(-1,-1)-4 \operatorname{Li}_{1,2}(1,-1)-4 \operatorname{Li}_{2,1}(1,-1) .
\end{aligned}
$$

The terms involving multiple polylogarithms of length greater than 1 may be expressed as terms involving ordinary polylogarithms (of length 1). First, we reduce the multiple polylogarithms from length 3 to length 2 and 1 using the following identities:

$$
\begin{aligned}
\operatorname{Li}_{1,1,1}(-1,-1,-1)= & \frac{1}{3}\left(\operatorname{Li}_{1}(-1) \operatorname{Li}_{1,1}(-1,-1)-\operatorname{Li}_{2,1}(1,-1)-\operatorname{Li}_{1,2}(-1,1)\right), \\
\operatorname{Li}_{1,1,1}(1,-1,-1)= & \frac{1}{12}\left(6 \operatorname{Li}_{1}(-1) \operatorname{Li}_{1,1}(1,-1)-2 \operatorname{Li}_{1}(-1) \operatorname{Li}_{1,1}(-1,-1)\right. \\
& -\operatorname{Li}_{2,1}(1,-1)-\operatorname{Li}_{1,2}(-1,1) \\
& \left.-6 \operatorname{Li}_{2,1}(-1,-1)-6 \operatorname{Li}_{1,2}(1,1)\right), \\
\operatorname{Li}_{1,1,1}(1,1,-1)= & \frac{1}{6}\left(\operatorname{Li}_{1}(-1)\right)^{3}, \\
\operatorname{Li}_{1,1,1}(-1,1,-1)= & \frac{1}{6}\left(2 \operatorname{Li}_{1}(-1) \operatorname{Li}_{1,1}(-1,-1)+\operatorname{Li}_{2,1}(1,-1)+\operatorname{Li}_{1,2}(-1,1)\right) .
\end{aligned}
$$

Incorporating these identities in the expression for $I$, we get

$$
\begin{aligned}
I= & \frac{2}{3} \operatorname{Li}_{1}(-1) \operatorname{Li}_{1,1}(-1,-1)-\frac{2}{3} \operatorname{Li}_{2,1}(1,-1)-\frac{2}{3} \operatorname{Li}_{1,2}(-1,1) \\
& +\operatorname{Li}_{1}(-1) \operatorname{Li}_{1,1}(1,-1)-\frac{1}{3} \operatorname{Li}_{1}(-1) \operatorname{Li}_{1,1}(-1,-1)-\frac{1}{6} \operatorname{Li}_{2,1}(1,-1) \\
& -\frac{1}{6} \operatorname{Li}_{1,2}(-1,1)-\mathrm{Li}_{2,1}(-1,-1)-\mathrm{Li}_{1,2}(1,1)+\frac{1}{3}\left(\operatorname{Li}_{1}(-1)\right)^{3} \\
& +\frac{2}{3} \operatorname{Li}_{1}(-1) \operatorname{Li}_{1,1}(-1,-1)+\frac{1}{3} \operatorname{Li}_{2,1}(1,-1)+\frac{1}{3} \operatorname{Li}_{1,2}(-1,1) \\
& -4 \operatorname{Li}_{1,2}(-1,-1)-4 \operatorname{Li}_{2,1}(-1,-1)-4 \operatorname{Li}_{1,2}(1,-1)-4 \mathrm{Li}_{2,1}(1,-1) \\
= & \mathrm{Li}_{1}(-1) \operatorname{Li}_{1,1}(-1,-1)-\frac{9}{2} \operatorname{Li}_{2,1}(1,-1)-\frac{1}{2} \operatorname{Li}_{1,2}(-1,1) \\
& +\operatorname{Li}_{1}(-1) \operatorname{Li}_{1,1}(1,-1)-5 \operatorname{Li}_{2,1}(-1,-1)-\operatorname{Li}_{1,2}(1,1) \\
& +\frac{1}{3}\left(\operatorname{Li}_{1}(-1)\right)^{3}-4 \operatorname{Li}_{1,2}(-1,-1)-4 \operatorname{Li}_{1,2}(1,-1) .
\end{aligned}
$$

Now we consider identities for multiple polylogarithms for length 2 in terms of classical polylogarithms:

$$
\begin{aligned}
\operatorname{Li}_{1,1}(-1,-1) & =\frac{1}{2}\left(\left(\operatorname{Li}_{1}(-1)\right)^{2}-\operatorname{Li}_{2}(1)\right), \\
\operatorname{Li}_{2,1}(1,-1) & =-\frac{1}{4}\left(2 \operatorname{Li}_{2}(1) \operatorname{Li}_{1}(-1)+\operatorname{Li}_{3}(1)\right), \\
\operatorname{Li}_{1,2}(-1,1) & =\frac{1}{2}\left(3 \operatorname{Li}_{2}(1) \operatorname{Li}_{1}(-1)+2 \operatorname{Li}_{3}(1)\right), \\
\operatorname{Li}_{1,1}(1,-1) & =\frac{1}{2}\left(\operatorname{Li}_{1}(-1)\right)^{2},
\end{aligned}
$$




$$
\begin{aligned}
\operatorname{Li}_{2,1}(-1,-1) & =\frac{1}{8}\left(8 \mathrm{Li}_{2}(1) \mathrm{Li}_{1}(-1)+5 \mathrm{Li}_{3}(1)\right) \\
\operatorname{Li}_{1,2}(1,1) & =\mathrm{Li}_{3}(1) \\
\mathrm{Li}_{1,2}(-1,-1) & =\frac{1}{8}\left(-12 \mathrm{Li}_{2}(1) \operatorname{Li}_{1}(-1)-13 \mathrm{Li}_{3}(1)\right) \\
\mathrm{Li}_{1,2}(1,-1) & =\frac{1}{8} \mathrm{Li}_{3}(1)
\end{aligned}
$$

Applying the previous identities to the expression for $I$ gives

$$
\begin{aligned}
I= & \frac{1}{2}\left(\operatorname{Li}_{1}(-1)\right)^{3}-\frac{1}{2} \operatorname{Li}_{2}(1) \operatorname{Li}_{1}(-1)+\frac{9}{4} \mathrm{Li}_{2}(1) \mathrm{Li}_{1}(-1)+\frac{9}{8} \mathrm{Li}_{3}(1) \\
& -\frac{3}{4} \mathrm{Li}_{2}(1) \mathrm{Li}_{1}(-1)-\frac{1}{2} \mathrm{Li}_{3}(1)+\frac{1}{2}\left(\mathrm{Li}_{1}(-1)\right)^{3} \\
& -5 \mathrm{Li}_{2}(1) \mathrm{Li}_{1}(-1)-\frac{25}{8} \mathrm{Li}_{3}(1)-\mathrm{Li}_{3}(1) \\
& +\frac{1}{3}\left(\mathrm{Li}_{1}(-1)\right)^{3}+6 \mathrm{Li}_{2}(1) \mathrm{Li}_{1}(-1)+\frac{13}{2} \mathrm{Li}_{3}(1)-\frac{1}{2} \mathrm{Li}_{3}(1) \\
= & \frac{4}{3}\left(\mathrm{Li}_{1}(-1)\right)^{3}+2 \mathrm{Li}_{2}(1) \mathrm{Li}_{1}(-1)+\frac{5}{2} \mathrm{Li}_{3}(1) .
\end{aligned}
$$

We may now write the expression in terms of values of the zeta function and logarithms:

$$
I=-\frac{4}{3} \log ^{3} 2-2 \zeta(2) \log 2+\frac{5}{2} \zeta(3)
$$

This shows the required identity for the formula (5).

The previous theorem may be completed with the trivial statement

$$
m(x+y+2)=\log 2 .
$$

In fact, the motivation for setting $c=2$ is that this is the precise point where the family of polynomials $x+y+c$ reaches the unit torus singularly. In classical Mahler measure, those polynomials are among the simplest to compute the Mahler measure, and the same is true in higher Mahler measures.

6.4. A family related with Dyson integrals. Consider the family of polynomials

$$
\begin{aligned}
P_{N}\left(x_{1}, \ldots, x_{N}\right) & =\prod_{1 \leq h \neq j \leq N}\left(1-\frac{x_{h}}{x_{j}}\right)=\prod_{h<j}\left(2-\frac{x_{h}}{x_{j}}-\frac{x_{j}}{x_{h}}\right) \\
& =2^{N(N-1)} \prod_{h<j} \sin ^{2} \pi\left(\theta_{h}-\theta_{j}\right), \quad x_{h}=e^{2 \pi i \theta_{h}} .
\end{aligned}
$$

Then we have the following result, due to Dyson:

$$
Z\left(k, P_{N}\right)=\int_{0}^{1} \cdots \int_{0}^{1} P_{N}\left(e^{2 \pi i \theta_{1}}, \ldots, e^{2 \pi i \theta_{N}}\right)^{k} d \theta_{1} \cdots d \theta_{N}=\frac{(N k) !}{(k !)^{N}} .
$$


Incorporating this identity into the formula for the zeta Mahler measure we obtain

$$
\begin{aligned}
Z\left(s, 1+\lambda P_{N}\right) & =\int_{0}^{1} \cdots \int_{0}^{1}\left(1+\lambda P_{N}\right)^{s} d \theta_{1} \cdots d \theta_{N} \\
& =\sum_{k=0}^{\infty}\left(\begin{array}{l}
s \\
k
\end{array}\right) Z\left(k, P_{N}\right) \lambda^{k}=\sum_{k=0}^{\infty}\left(\begin{array}{l}
s \\
k
\end{array}\right) \frac{(N k) !}{(k !)^{N}} \lambda^{k} \\
& ={ }_{N} F_{N-1}\left(\begin{array}{c}
-s, \frac{1}{N}, \frac{2}{N}, \ldots, \frac{N-1}{N} \\
1, \ldots, 1
\end{array} \mid \frac{\lambda}{N^{N}}\right) .
\end{aligned}
$$

As always, we may use the expression of zeta to compute higher Mahler measures. By Lemma 18(ii),

$$
\begin{aligned}
m\left(1+\lambda P_{N}\right) & =\sum_{k=1}^{\infty} \frac{(-1)^{k-1}}{k} Z\left(k, P_{N}\right) \lambda^{k}=\sum_{k=1}^{\infty} \frac{(-1)^{k-1}}{k} \frac{(N k) !}{(k !)^{N}} \lambda^{k} \\
m_{2}\left(1+\lambda P_{N}\right) & =\sum_{k=1}^{\infty} \frac{(-1)^{k}}{k}\left(1+\cdots+\frac{1}{k-1}\right) Z\left(k, P_{N}\right) \lambda^{k} \\
& =\sum_{k=1}^{\infty} \frac{(-1)^{k}}{k}\left(1+\cdots+\frac{1}{k-1}\right) \frac{(N k) !}{(k !)^{N}} \lambda^{k} .
\end{aligned}
$$

In particular, for $N=2$,

$$
\begin{aligned}
m\left(1+\lambda P_{2}\right) & =\sum_{k=1}^{\infty} \frac{(-1)^{k-1}}{k}\left(\begin{array}{c}
2 k \\
k
\end{array}\right) \lambda^{k}, \\
m_{2}\left(1+\lambda P_{2}\right) & =\sum_{k=2}^{\infty} \frac{(-1)^{k}}{k}\left(1+\cdots+\frac{1}{k-1}\right)\left(\begin{array}{c}
2 k \\
k
\end{array}\right) \lambda^{k} .
\end{aligned}
$$

These correspond to the higher Mahler measures of $1+\lambda\left(x+x^{-1}+y+y^{-1}\right)$.

Acknowledgements. We would like to thank Fernando RodríguezVillegas for helpful discussions.

\section{References}

[1] H. Akatsuka, Zeta Mahler measures, in: Mahler measure conference, Tokyo Institute of Technology, December 2007.

[2] D. J. Broadhurst, Massive 3-loop Feynman diagrams reducible to $\mathrm{SC}^{*}$ primitives of algebras of the sixth root of unity, Eur. Phys. J. C Part. Fields 8 (1999), 313-333.

[3] Y. Ohno and D. Zagier, Multiple zeta values of fixed weight, depth, and height, Indag. Math. (N.S.) 12 (2001), 483-487. 
[4] F. Rodríguez-Villegas, Modular Mahler measures I, in: Topics in Number Theory (University Park, PA, 1997), Math. Appl. 467, Kluwer, Dordrecht, 1999, 17-48.

[5] - personal communication, August 2007.

[6] C. J. Smyth, On measures of polynomials in several variables, Bull. Austral. Math. Soc. Ser. A 23 (1981), 49-63; Corrigendum (with G. Myerson), ibid. 26 (1982), 317319.

Department of Mathematics

Tokyo Institute of Technology

2-12-1 Oh-Okayama, Meguro

Tokyo 152-8551, Japan

E-mail: kurokawa@math.titech.ac.jp

Department of Mathematics

Nagoya University

Furo, Chikusa

Nagoya 464-8602, Japan

E-mail: ochiai@math.nagoya-u.ac.jp
Department of Mathematical and Statistical Sciences University of Alberta Edmonton, AB T6G 2G1, Canada E-mail: mlalin@math.ualberta.ca

Received on 26.4.2008

and in revised form on 16.8.2008 